\title{
Interfacial structure of $70 \%$ fish oil-in-water emulsions stabilized with combinations of sodium caseinate and phosphatidylcholine
}

Yesiltas, Betül; Torkkeli, Mika; Almásy, László; Dudás, Zoltán; Wacha, András Ferenc; Dalgliesh, Robert; García-Moreno, Pedro Jesús; Moltke Sørensen, Ann Dorit ; Jacobsen, Charlotte; Knaapila, Matti

Published in:

Journal of Colloid and Interface Science

Link to article, DOI:

10.1016/j.jcis.2019.06.103

Publication date:

2019

Document Version

Peer reviewed version

Link back to DTU Orbit

Citation (APA):

Yesiltas, B., Torkkeli, M., Almásy, L., Dudás, Z., Wacha, A. F., Dalgliesh, R., García-Moreno, P. J., Moltke Sørensen, A. D., Jacobsen, C., \& Knaapila, M. (2019). Interfacial structure of $70 \%$ fish oil-in-water emulsions stabilized with combinations of sodium caseinate and phosphatidylcholine. Journal of Colloid and Interface Science, 554, 183-190. https://doi.org/10.1016/j.jcis.2019.06.103

\section{General rights}

Copyright and moral rights for the publications made accessible in the public portal are retained by the authors and/or other copyright owners and it is a condition of accessing publications that users recognise and abide by the legal requirements associated with these rights.

- Users may download and print one copy of any publication from the public portal for the purpose of private study or research.

- You may not further distribute the material or use it for any profit-making activity or commercial gain

- You may freely distribute the URL identifying the publication in the public portal 
1

\section{Interfacial structure of $70 \%$ fish oil-in-water emulsions}

\section{stabilized with combinations of sodium caseinate and}

\section{phosphatidylcholine}

Betül Yesiltas, ${ }^{1, *}$ Mika Torkkeli, ${ }^{2, *}$ László Almásy, ${ }^{3,4}$ Zoltán Dudás, ${ }^{3}$ András Ferenc Wacha, ${ }^{5}$ Robert

Dalgliesh, ${ }^{6}$ Pedro J. García-Moreno, ${ }^{1}$ Ann-Dorit M. Sørensen, ${ }^{1}$ Charlotte Jacobsen, ${ }^{1, *}$ and Matti $\mathrm{Knaapila}^{2, *}$

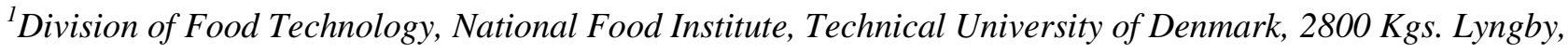

Denmark, ${ }^{2}$ Department of Physics, Technical University of Denmark, 2800 Kgs. Lyngby, Denmark, ${ }^{3}$ Institute for Solid State Physics and Optics, Wigner Research Centre for Physics, 1525 Budapest, Hungary, ${ }^{4}$ State Key Laboratory of Environment-Friendly Energy Materials, Southwest University of Science and Technology, Mianyang 621010, China, ${ }^{5}$ Institute of Materials and Environmental Chemistry, Research Centre for Natural Sciences Hungarian Academy of Sciences, 1525 Budapest, Hungary, ${ }^{6}$ Rutherford Appleton Laboratory, ISIS Facility, Chilton, OX11 OQX, UK

*Corresponding authors: betye@food.dtu.dk, mtork@fysik.dtu.dk, chja@food.dtu.dk, matti.knaapila@fysik.dtu.dk 


\begin{abstract}
We report on the structural evaluation of high fat fish oil-in-water emulsions emulsified with sodium caseinate (CAS) and phosphatidylcholine (PC). The microemulsions contained 70\% (w/w) fish oil with $1.05-1.4 \%(\mathrm{w} / \mathrm{w})$ CAS and $0.4-1.75 \%(\mathrm{w} / \mathrm{w})$ PC and were studied by the combination of light scattering together with small-angle X-ray and neutron scattering (SAXS/SANS). Aqueous CAS forms aggregates having a denser core of about $100 \mathrm{kDa}$ and less dense shell about $400 \mathrm{kDa}$ with the hard sphere diameter of $20.4 \mathrm{~nm}$. PC appears as multilayers whose coherence length spans from 40 to 100 nm. PC monolayer separates oil and water phases. Moreover, 80\% CAS particles are loosely bound to the interface but are not forming continuous coverage. The distance between aggregated CAS particles in microemulsion is increased compared to CAS aggregates in pure CAS-in-water system. PC multilayers become larger in the presence of oil-water interface compared to the pure PC mixtures. Bilayers become larger with increasing PC concentration. This study forms a structural base for the combination of CAS and PC emulsifiers forming a well-defined thin and dense PC layer together with thick but less dense CAS layer, which is assumed to explain its better oxidative stability compared to single emulsifiers.
\end{abstract}

Keywords: interfacial structure, emulsifier adsorption, high fat omega-3 delivery emulsions, SAXS, SANS 


\section{Introduction}

Marine long chain (LC) omega-3 polyunsaturated fatty acids (PUFAs) have myriad beneficial effects on health such as decreasing risk of cardiovascular diseases, reducing inflammation and even play an important role in mental health $[1,2,3,4]$. Fish oil-in-water emulsions can be used as delivery systems to enrich foods with LC omega-3 PUFAs including eicosapentaenoic (C20:5n- 3, EPA) and docosahexaenoic (C22:6n-3, DHA) acids [5]. High fat omega-3 delivery emulsions have further advantages, when it comes to enrichment of food systems with high viscosity or high fat content (e.g., mayonnaise, dressings, and cream cheese), such as the fact that addition of relatively lower amount of delivery emulsion is necessary for enrichment. Moreover, similar texture/structure of the delivery emulsion and food system provides easiness for mixing these two systems [6, 7].

However, delivery of bioactive compounds comes with its challenges in terms of physical and oxidative stability. LC omega-3 PUFAs are highly prone to oxidation, which results in formation of lipid oxidation products causing undesired sensory properties as well as loss in nutritional profile. Moreover, delivery systems need to be physically stabilized using the right concentration and combination of emulsifiers as well as improving other conditions during emulsion production. Many factors play important roles for the physical and oxidative stability of these emulsions, such as ingredients (type, quality, and amount), $\mathrm{pH}$, temperature (during production and storage), homogenizers (type and conditions), droplet size, viscosity and surface charge [8, 9]. These factors also have an effect on the formation and properties of the oil-water interface in high fat oil-in-water emulsions. Previous research has shown that lipid oxidation in emulsions are initiated at the oil-water interface and, therefore, food researchers and industry are highly interested in characterizing the oilwater interface and understanding the inner dynamics of these systems. 
The stabilization against oxidation depends strongly on the oil-water interface of oil-in-water emulsions. The protein-stabilized interfaces are less efficient in protecting emulsified lipids against oxidation than surfactant-stabilized interfaces [10]. Combined use of protein (CAS or whey protein) and surfactants (PC, Tween 60, or lecithin) increases oxidative stability of the fish oil-in-water emulsions $[11,12,13,14]$.

Particular attention has been placed on combination of CAS and surfactants and adsorption and partitioning of these emulsifiers. It is expected that surfactants alter the characteristics of adsorbed CAS. For example, lecithin may adsorb to the available hydrophobic areas at the interface where there is a bare fat surface in between CAS molecules and this prevents aggregation and coalescence of the lipid droplets $[12,13]$. It is also known that at higher levels of phospholipid concentrations, the lower limit of CAS layer thickness increases from 5 to $6.5 \mathrm{~nm}$ while the upper plateau limit decreases from 10 to $8 \mathrm{~nm}$ [12]. The presence of egg-PC or 1,2-di-(9Z-octadecenoyl)-sn-glycero-3-phosphocholine (DOPC) in the casein emulsions, gives a thicker CAS layer at low CAS concentration $(<1 \%)$ and a thinner layer at higher concentrations $(>1 \%)$ [15]. Increase in the layer thickness is attributed to the change in packing of the adsorbed CAS molecules with phospholipids co-adsorbed at the interface, which was explained by the lack of necessity for CAS to extend so far to cover the oil-water interface, instead the adsorbed molecule adopt a more favored structure [15]. Surfactants may not only displace protein from the interface but also bind to proteins and alter their conformation [16,17]. The structure of oil-water interface is not necessarily homogenous, potentially having partly separated domains. Flexible, disordered proteins such as CAS form thicker but less dense interfacial layers [18]. Surfactant molecules with high surface activity may then adsorb to the interface through the gaps in between protein molecules [19]. 
Investigating structural details of CAS with and without surfactants has significant impact on understanding the ability of controlling lipid oxidation in oil-in-water emulsions, as these affect parameters such as; surface charge, thickness, and structure of oil-water interface. While the colloidal structures of different forms of CAS have been thoroughly studied by neutrons and X-rays [20, 21, 22], similar studies of oil-water interfaces consisting of combination of emulsifiers are less comprehensive.

In this paper, we use a combination of X-ray and neutron scattering to investigate the structure of a specific $70 \%$ fish oil-in-water emulsion produced with combined use of CAS and PC emulsifiers. As deuterated fish oil is not obtainable, and as fish oil has strong incoherent scattering background in neutron experiments, some of the experiments were carried out by replacing fish oil by deuterated hexadecane. This choice of hexadecane was motivated by the fact that fish oil is dominated by long chain fatty acids. Furthermore, hexadecane is well described in various oil-in-water emulsions (see, e.g., Rampon et al. [23]). Our results suggest that the interface incorporates a thin PC layer while $80 \%$ of CAS molecules remain loosely bound to this interface. Presence of oil-water interface promotes PC multilayers and affects period between PC surfactants in the aqueous phase in the vicinity of the interface. At the same time the presence of interface affects the distance between CAS molecules within CAS aggregates. This is the first study where SANS and SAXS techniques are applied in high fat fish oil-in-water emulsions. 


\section{Materials and Methods}

\subsection{Materials}

Cod liver oil was provided by Maritex A/S, subsidiary of TINE, BA (Sortland, Norway), and stored at $40^{\circ} \mathrm{C}$ until use. The fatty acid (\% of total fatty acids) content of the fish oil as determined and supplied by the manufacturer was as follows: C14:0 (3.02), C16:0 (8.91), C16:1n-7 (8.20), C18:0 (1.88), C18:1n-9 (16.00), C18:1n-7 (5.16), C18:2n-6 (1.79), C18:3n-3 (0.84), C20:1n-9 (11.59), C20:5n-3 (9.27), C22:1n-11 (6.06), C22:6n-3 (11.64) and other fatty acids (15.64). Sodium caseinate, CAS, (Miprodan 30) was donated by Arla Foods Ingredients amba (Viby J, Denmark). Arla reported a protein content of $92 \%$ in CAS for Miprodan 30. PC from soybean (LIPOID S 100) was donated by Lipoid GmbH (Ludwigshafen, Germany). Specifications from Lipoid reported that phosphatidylcholine content was $96.2 \%$ for LIPOID S 100 . Deuterium oxide $\left(\mathrm{D}_{2} \mathrm{O}\right)(98 \% \mathrm{D})$ and deuterated hexadecane $(n$ hexadecane- $\left.\mathrm{d}_{34}\right)(98 \% \mathrm{D})$ were purchased from Chemtronica (Stockholm, Sweden). Hexadecane ( $n$ hexadecane) was purchased from Bie\&Berntsena-S (Denmark).

\subsection{Sample preparation}

Pure compounds (CAS and PC) were dissolved/dispersed in $\mathrm{D}_{2} \mathrm{O}$ for SANS and $\mathrm{H}_{2} \mathrm{O}$ for SAXS experiments under magnetic stirring $(500 \mathrm{rpm})$ overnight. The concentrations of the CAS and PC samples were ranged between $1-10 \%(\mathrm{w} / \mathrm{w})$ and $0.4-6 \%(\mathrm{w} / \mathrm{w})$, respectively.

Aqueous phases of the emulsions were prepared one day before and left overnight on the magnetic stirrer $(500 \mathrm{rpm})$ in order to allow the CAS $(1.05 \%, \mathrm{w} / \mathrm{w})$ and $\mathrm{PC}(1.75 \%, \mathrm{w} / \mathrm{w})$ to dissolve in $\mathrm{D}_{2} \mathrm{O}$ or $\mathrm{H}_{2} \mathrm{O}$ at room temperature [24]. Emulsions (10g) were prepared using hand-held ultraturrax (POLYTRON PT 1200 E). Water phase was mixed for $30 \mathrm{~s}$ using hand-held ultraturrax and oil phase 
$(70 \%, w / w)$ was added slowly in 3 min while the aqueous phase was continuously mixed. After adding oil phase, emulsion was mixed for additional 4 min. Measurements were done within 3 days after sample preparation. There were no added buffer and the sample $\mathrm{pH}$ was 6.86 . The details of prepared samples are listed in Tables S1 and S2 in the Supplementary Material.

\subsection{Droplet size}

Particle size of the emulsions was measured by laser diffraction (Mastersizer 2000, Malvern Instruments Ltd., Worcestershire, UK) using the method described by Yesiltas et al. [5]. Emulsion (1 g) was dissolved in $5 \mathrm{~g}$ sodium dodecyl sulfonate (SDS) buffer (10 mM NaH2PO4, $5 \mathrm{mM} \mathrm{SDS}, \mathrm{pH}$ 7), mixed for $30 \mathrm{~s}$ and then sonicated for $15 \mathrm{~min}$ in a water bath. Droplets of the pretreated emulsions were diluted in recirculating water (3000 rpm), reaching an obscuration of $12-15 \%$. The refractive indices of sunflower oil (1.469) and water (1.330) were used for particle and dispersant.

\subsection{Relative protein content in the aqueous phase}

Protein determination in the aqueous phase of emulsions was done mainly based on the method described by Jacobsen, Meyer, \& Adler-Nissen [25]. Emulsions ( $20 \mathrm{~g})$ were centrifuged for $10 \mathrm{~min}$ at $25400 \mathrm{~g}$ and $10{ }^{\circ} \mathrm{C}$ (Sorvall RC-6 PLUS, Thermo Fisher Scientific, Osterode, Germany; rotor SS-34) and the oil phase was removed by the use of a pipette. The rest was mixed with distilled water (1:2) and then subjected to ultracentrifugation (Beckman Ultracentrifuge L8-60M, Fullerton, CA; rotor 21102) for $16 \mathrm{~h}$ at $106979 \mathrm{~g}$ and $15{ }^{\circ} \mathrm{C}$, and once again the aqueous phase was extracted by the use of a syringe. The aqueous phase was diluted 1:6 in distilled water and protein concentration was determined using a BCA protein assay reagent kit (Pierce, Thermo Scientific, Rockford, IL, USA) by measuring in a spectrophotometer (Shimadzu, UV mini 1240, Kyoto, Japan) at $562 \mathrm{~nm}$. Results were presented as 
relative protein content in the aqueous phase, which was obtained by calculating the percentage of protein content in the aqueous phase compared to total protein amount added into the emulsion.

\subsection{SAXS}

SAXS measurements on the emulsions were done on the CREDO apparatus of the Research Centre for Natural Sciences, Hungarian Academy of Sciences [26, 27]. Cu Ka X-rays produced by a GeniX3D Cu ULD integrated beam delivery system (Xenocs SA, Sassenage, France), and the scattered radiation was detected with a Pilatus-300k CMOS hybrid pixel position sensitive detector (Dectris Ltd., Baden, Switzerland) placed $536 \mathrm{~mm}$ downstream from the sample. The samples were filled into borosilicate glass capillaries of approx. $1 \mathrm{~mm}$ outer diameter and $0.01 \mathrm{~mm}$ wall thickness and kept at $25^{\circ} \mathrm{C}$. The $q$ range was 0.2 to $3 \mathrm{~nm}^{-1}$ calibrated by SBA15 mesoporous silica. The total exposure time was divided into 5 minute units, which were repeated several times until the desired signal-to-noise ratio was obtained. Each scattering pattern was corrected for sample self-absorption, instrumental background and detector flatness using the standard procedure implemented in the data collection program. The scattering intensity was transformed to absolute units (differential scattering cross section) by measuring a glassy carbon sample of known absolute scattering intensity under the same conditions as our samples and the scattering patterns were statistically filtered to remove artefacts from external radiation. 


\subsection{SANS}

SANS measurements were performed on the Yellow Submarine instrument at the BNC in Budapest (Hungary) and the Larmor Instrument, Rutherford Appleton Laboratory (Chilton, England). At the Yellow Submarine, the wavelengths of 4.38 and $10.23 \AA$ were used at the sample-to-detector distances of 1.15 and $5.25 \mathrm{~m}$ leading to the overall $q$-range from 0.08 to $4 \mathrm{~nm}^{-1}$. Larmor was operated in the conventional SANS mode with the beam centered in the middle of the detector and a wavelength range of $0.09-1.25 \mathrm{~nm}$ leading to the overall $q$-range from 0.03 to $7 \mathrm{~nm}^{-1}$. In both cases, samples were filled in Hellma quartz cells of $2 \mathrm{~mm}$ path length and placed in the instrument and the measurements done at $25^{\circ} \mathrm{C}$. The measurement times were from 20 to 60 min. Empty cell and $\mathrm{H}_{2} \mathrm{O}$ were also measured. The scattering from the cell and solvent were subtracted from the data. 


\subsection{Analysis of small-angle scattering data}
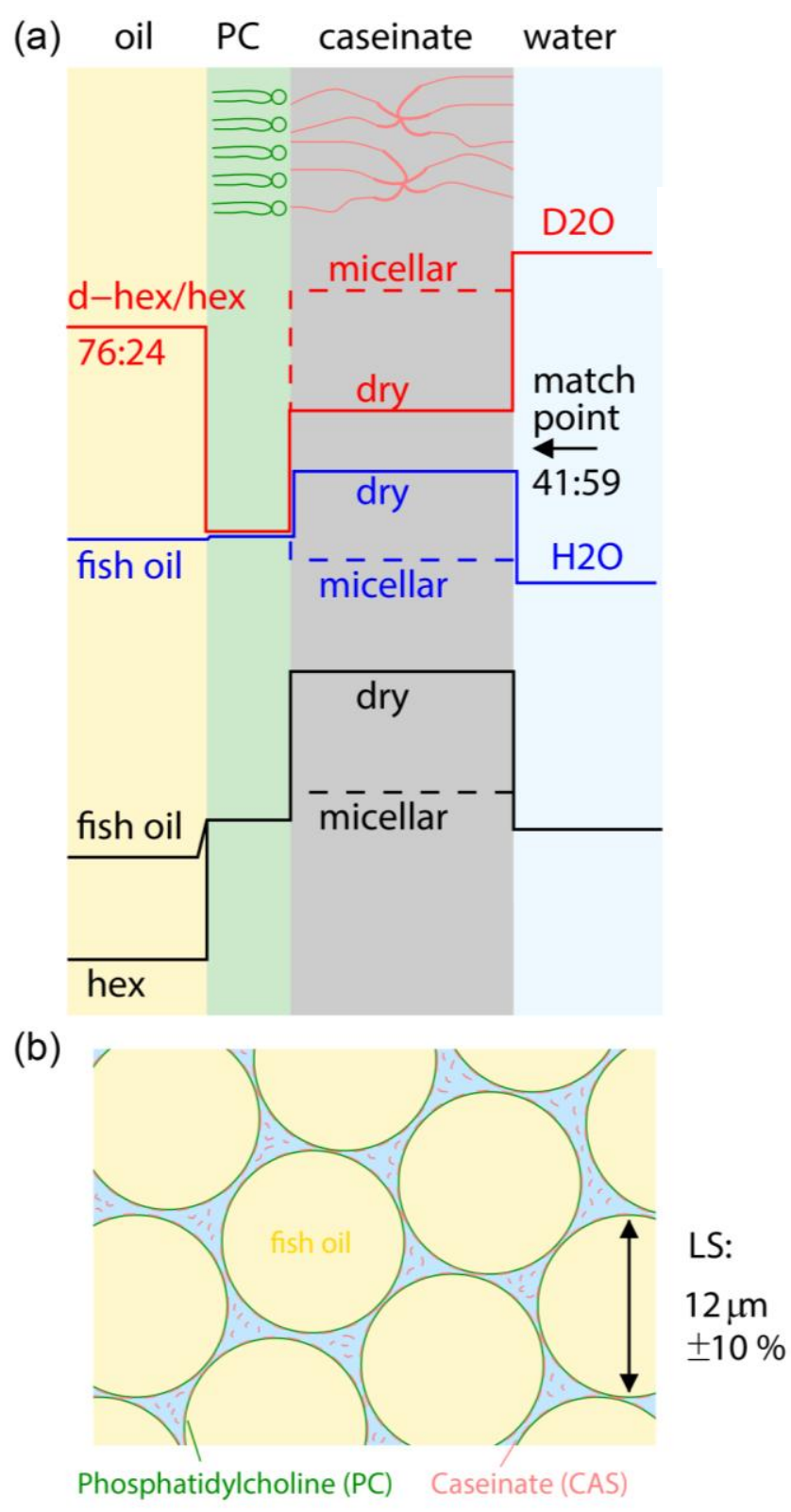
Fig. 1(a) illustrates the relative scattering length densities (SLDs) for the studied materials. Fig. 1(b) illustrates the apparent distribution of emulsion components. The exact SLD values for the employed samples are listed in Table S3 in the Supplementary Material. The scattering curves were first qualitatively discussed in terms of power law, as shown in Equation 1;

$I(q) \propto q^{-\alpha}$,

where $-\alpha$ is characteristic to the surface properties or to the way of agglomeration of scatterers.

The scattering intensity of CAS dissolved in water $\left(\right.$ or $\left.\mathrm{D}_{2} \mathrm{O}\right)$ were subsequently analyzed in order to model the contribution of the non-adsorbed CAS in the emulsion water phases. In our notation the CAS particles were assumed to be particles composed of a number of individual protein chains. For $q<0.451 / \mathrm{nm}$ the data were modelled in terms of core-shell type particles, whose interparticle interference is given by Percus-Yevick (P-Y) structure factor $S_{P Y}\left(q, R_{H S}, \eta\right)$ for hard spheres [28]. The intensity was understood as shown in Equation 2;

$I(q)=n\left(V_{p} \Delta \rho\right)^{2}\left[\left\langle F^{2}\right\rangle+\langle F\rangle^{2}\left(S_{P Y}-1\right)\right]$

where $n$ is the number density of particles, $V_{P}$ is the total volume of protein in the particle, $\Delta \rho$ is the scattering contrast between protein and water and $\left\langle F^{2}\right\rangle$ and $\langle F\rangle$ are the particle form factor and scattering amplitude, both normalized to one. The parameters of P-Y function are the hard sphere radius $R_{H S}$ and the particle volume fraction $\eta$. At high $\eta$, a peak develops at $q=2 \pi / D$, where $D=2 R_{H S}$ may be interpreted as particle diameter and also the distance between particles in a condensed system. 
For possible aggregation, this model was complemented as a background contribution as shown in Equation 3;

$I_{b g}=$ const. $q^{-\alpha}$

The scattering intensities of emulsions were understood as shown in Equation 4;

$I(q)=\frac{S}{V}\left[\left(\rho_{e}-\rho_{o}\right)^{2}+\left(\rho_{e}-\rho_{w}\right)^{2}-2\left(\rho_{e}-\rho_{o}\right)\left(\rho_{e}-\rho_{w}\right) \cos (q T)\right] \frac{2 \pi}{q^{4}}$,

where $\rho_{e}, \rho_{o}$ and $\rho_{w}$ are scattering length densities of emulsion (interface), oil and water where and $S / V$ is the specific surface area. $T$ is the film thickness at the oil-water interface. 


\section{Results and discussion}

\subsection{Pure compounds}

\subsubsection{CAS in water}
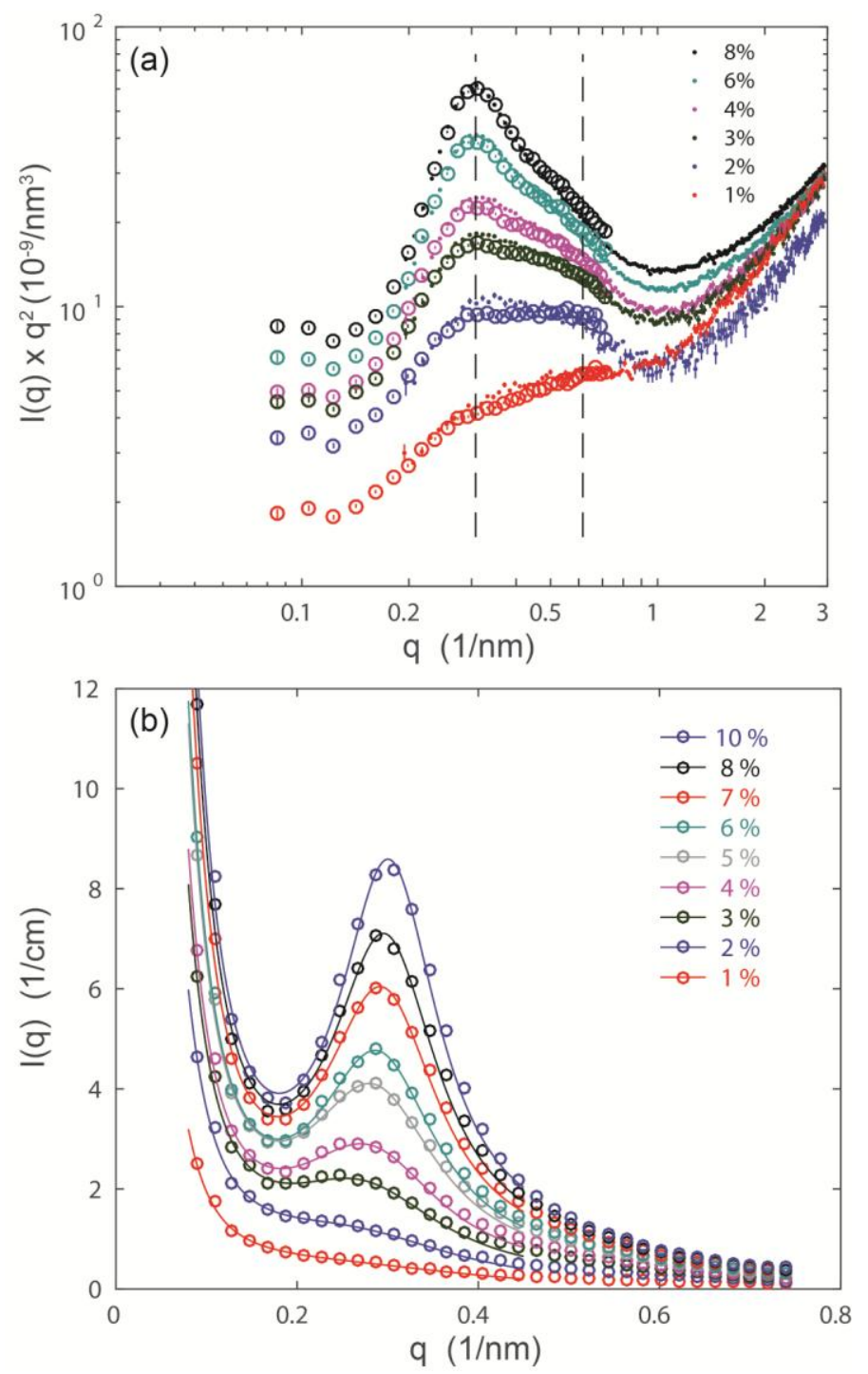

Fig. 2. (a) SAXS (solid lines) and SANS (circles) patterns of CAS solutions in $\mathrm{H}_{2} \mathrm{O}$ and $\mathrm{D}_{2} \mathrm{O}$, respectively, for various concentrations (Each SAXS intensity normalized to agree with respective SANS curve.) Dashed vertical lines mark positions of $1^{\text {st }}$ and $2^{\text {nd }}$ order maxima for periodicity $D=20.4 \mathrm{~nm}$ (b) SANS patters (circles) with the corresponding fits to the Eq. 2 and Eq. 3 (solid lines). The CAS concentration varies from $1 \%, \mathrm{w} / \mathrm{w}$ to $10 \%, \mathrm{w} / \mathrm{w}$. 
Fig. 3. Schematic illustration of the structure of studied CAS aggregates in water.

We note that the properties of CAS depend strongly on manufacturer, counterions, and counterion concentration etc. (See for example Smialowska et al. [29]). This motivates a careful structural consideration of the here employed CAS and the pure CAS-in-water systems before moving to the emulsions.

Fig. 2 shows the scattering data of CAS-in-water systems. Fig. 3 illustrates the discussed system. The fitting parameters are compiled in Table S4 in the Supplementary Material. Fig. 2 shows the effect of CAS concentration on the formation of aggregates. Both SAXS and SANS agree with the formation of uniform size aggregates, whose number increases linearly with concentration. In this paper, we denote the observed units in water as particles and their clusters as aggregates, although the clusters containing several CAS molecules are sometimes called micelles and the CAS molecules within these clusters as submicelles (see e.g., [22]).

As the mass and composition (water content) of these particles were not known exactly, it was not possible to use a priori known values for $\eta$. Moreover, the parameter $\eta$ does not grow linearly with CAS mass fraction, which indicates that the hard sphere model does not describe realistically the ordering of soft particles in dense CAS solutions (Fig. S1, Supplementary Material). Therefore, the 
parameter $\eta$ was taken as a fitting parameter describing order in the system rather than an exact volume fraction of the particles.

Fig. 3 also illustrates the form factors employed in the fitting. The first form factor included a dense core with the core radius $R_{C}$. The second form factor included $N$ random walk chains linked at the center of the particle. This approximates $N$ individual protein chains covering the hard core. The best fit is achieved for $R_{C}=4 \mathrm{~nm}$. We also tried the core-shell model with the shell radius $R_{S}=R_{H S}$ but this did not provide a better fit (Table S4, Supplementary Material)

This model does not explain an upturn at smallest angles, which may be caused by aggregation of the particles (or elsewhere called as submicelles [22]) into larger units. For the fitting, this upturn was treated as background as shown in Eq. 3. We found the best fit for $-\alpha=-2$. This implies that the larger arrangements are 2-dimensional at least to the certain extent.

The diameter of the particles is taken as twice the hard sphere radius of the P-Y fit which levels off quite well at $20.4 \mathrm{~nm}$ above $3 \%$ concentration. Below this, the hard sphere radius becomes meaningless as the average distance between particles increases which causes a shift in the scattering maxima to a smaller angle. The difference in peak location was not attributed to the change in size but their packing, which indicated CAS aggregates were more closely packed at higher concentrations compared to lower concentrations. This could be explained by the loss of the water molecules in between CAS aggregates when the concentration of CAS was increased.

The molar mass are estimated from the Fig. S2 in the Supplementary Material. In this consideration molecule weight of CAS is estimated from normalized scattering curves, which are extrapolated to zero concentration for all $q$, thus setting $S_{P-Y} \rightarrow 1$. This consideration may not be highly accurate, because 
our instruments do not make it possible to measure sufficiently low $q$ values. For the latter, we may estimate from scattering data that the particles consist of a dense core of about $100 \mathrm{kDa}$ protein weight and the total protein mass in the particle is about $400-500 \mathrm{kDa}$.

Our ideas are consistent with earlier neutron observations of Stothart et al. [20], who studied CAS submicelles obtained from whole milk micelles by acidification using SANS in concentration $16 \mathrm{mg} / \mathrm{ml}$ in $0.7 \mathrm{M} \mathrm{NaCl}$ solution in $\mathrm{D}_{2} \mathrm{O}$. The authors observe both the radius of gyration $R_{g}=6.5 \mathrm{~nm}$ and molar mass $300 \mathrm{~kg} / \mathrm{mol}$, while we observe $R_{g}=16.5 \mathrm{~nm}$ and molar mass $=430 \mathrm{~kg} / \mathrm{mol}$. If we use the obtained hard sphere radius and the voluminosity $\mathrm{mg} / \mathrm{mL}$ (ca. $20 \%$ dry weight) observed by Stothart, the calculated molar mass is $540 \mathrm{~kg} / \mathrm{mol}$ per particle. Note that $10 \%$ concentration would infer $9 \mathrm{~mL} / \mathrm{g}$, which corresponds to $300 \mathrm{~kg} / \mathrm{mol}$. This is then the theoretical lower limit of the molecule size for which value the submicelles would fill the whole volume, i.e. $\eta=1$.

Our results also agree with the X-ray work of Kumosinski et al., who showed a core-shell model for the particle, where $21 \%$ of the molar mass resides in a dense core of radius $4.4 \mathrm{~nm}$ surrounded by less dense shell of radius $11.4 \mathrm{~nm}$ [21]. The total mass was $285 \mathrm{~kg} / \mathrm{mol}$. It was also reported previously that CAS forms aggregates at the size of $10-11 \mathrm{~nm}$ in radius, similar to submicelles formed by casein [30, 31]. On the other hand, larger sizes were also reported by Huppertz et al. that suspensions of CAS appear as particles with $R_{g} \sim 20 \mathrm{~nm}$ and hydrodynamic radius $\sim 10 \mathrm{~nm}[32,33]$.

\subsubsection{PC in water}

The small-angle scattering data of employed PC in water systems divided by the concentration of the PC in $\mathrm{H}_{2} \mathrm{O}$ or $\mathrm{D}_{2} \mathrm{O}$ are plotted in Fig. $\mathrm{S} 3$ in the Supplementary Material. All samples showed a $q^{-3}$ power law and Lorentzian shaped Bragg peaks that stem from the known layer-like structure and 
whose area increased with the increasing concentration [34]. Due to the higher resolution, we used Xray data for the line shape analysis. The long period corresponding to the thickness of PC bilayers and the coherence length $\xi$ corresponding to the thickness of the PC multilayers are compiled in Table 1 (vide infra). When the PC concentration was increased from $0.4 \%$ to $0.9 \%$, the bilayer thickness increased from 6.29 to $6.33 \mathrm{~nm}$, respectively. Calculated coherence lengths (size of the structures) were $97.51 \mathrm{~nm}$ and $87.54 \mathrm{~nm}$, respectively. This corresponds to ca. 15 bilayers.

\subsection{Oil-water interface of $70 \%$ oil-in-water emulsions}

\subsubsection{Droplet size}

The prepared high fat emulsions stabilized with CAS and PC contained $70.0 \%$ of oil and $27.2 \%$ of water. The samples appear milky having a lower viscosity than mayonnaise (70-80\% fat), but being more viscous than milk. Samples did not show any observable instability during the measurements. Droplet size of the emulsions produced with hexadecane or fish oil are shown in Table S5 in the Supplementary Material. The difference between their $\mathrm{D}[3,2]$ and specific surface area was not significant.

70\% fish oil-in-water emulsions with different combinations of CAS and PC were also analyzed in order to study differences between presence of both emulsifiers together, absence of one of the emulsifiers, and different concentrations of emulsifiers. Table S6 in the Supplementary Material shows the droplet size and specific surface area of these emulsions. Emulsion CAS/PC 1.05/1.75 had smaller droplets compared to emulsion with only CAS 1.05 which could be due to the contribution of PC at the oil-water interface. Emulsion CAS 2.8 had smaller droplets compared to emulsion CAS 1.05 due to the higher concentration of CAS as an emulsifier; smaller droplets also resulted in higher surface area. 
Large droplet sizes of emulsions PC 1.75 and PC 2.8 indicated that the emulsions were not as physically stable as emulsions produced with only CAS or combinations of CAS and PC. The estimated CAS contents left in the aqueous phase after emulsion preparation are also compiled in Table S6 in the Supplementary Material. The data show that replacement of CAS by PC increases the amount of CAS in the aqueous phase.

\subsubsection{SAXS and SANS}

Fig. 4 plots the SANS patterns of different oil-in-water emulsions with fish oil and deuterated hexadecane. The solid lines are expected total scattering from PC monolayer and CAS particles including constant background (vide infra). The dashed blue line (above) shows the calculated contribution of a single monolayer film in a nominally matched sample (deuterated hexadecane:hexadecane 0.945:0.055). The black dashed line (below) shows the contribution of CAS particles $(40 \%)$ alone. 


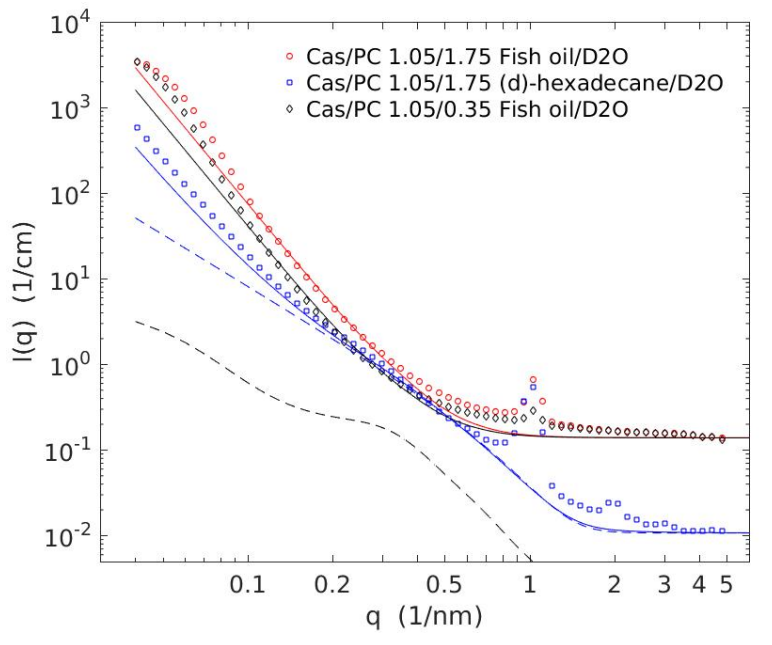

Fig. 4. SANS patterns (circles) for selected emulsions. The solid lines are expected total scattering from PC monolayer and CAS particles with a constant background contribution, while dashed lines represent the individual scattering contribution from CAS particles (black) and PC monolayer (blue). All material fractions are given in $\%, w / w$. See text for details.

The reasoning for the above-mentioned model (solid lines) is as follows. The scattering curves with fish oil are dominated by $q^{-4}$ slope which arises from globular particles. The scattering curve with deuterated hexadecane follows $q^{-2}$ slope. This means scattering comes from a thin film since the region only extends to $q \propto 1 / T$, where $T$ represents the interface thickness. This allows us to identify well defined layer with $T \approx 3.2 \mathrm{~nm}$. As this number corresponds to the PC monolayer, we propose that the interface contains a well-defined PC monolayer. Following this idea, fits to the PC monolayer to oil-water interface in the case of fish oil and deuterated hexadecane are shown in Fig. S4 in the Supplementary Material.

Calculations of scattering patterns are based on true densities where the specific surface $S / V$ is left as a free parameter. The curves furthermore include calculated incoherent background. In case of 
deuterated hexadecane, there is also calculated contribution from the molecular level inhomogenities that arise from mixing large deuterated and nondeuterated molecules. As hexadecane is a linear molecule, this leads to a $q^{-1}$ tail contribution which is calculated and detailed in Fig. S4 in the Supplementary Material. A similar tail may also be present in fish oil which is a mixture of different fatty acids in triglyceride form.

A thin film at oil-water interfacial area was estimated from Eq. 4. The value for $S / V$ was found to be $35001 / \mathrm{cm}$ for fish oil and $110001 / \mathrm{cm}$ for deuterated hexadecane. Using $S / V=6 \varphi / D_{\text {droplet }}$, the corresponding droplet diameters determined from SAXS and SANS are $D_{\text {droplet }}=12 \mu \mathrm{m}$ and $D_{\text {droplet }}=3.9 \mu \mathrm{m}$. This agrees with the droplet size measurements (Table S5, Supplementary Material) but also implies that fish oil and hexadecane may have slightly different droplet sizes.

In addition, the SANS curves include a broad feature at $0.2-0.5 \mathrm{~nm}^{-1}$ arising from CAS and Bragg peaks at about $1 \mathrm{~nm}^{-1}$ arising from PC (Fig. 4). The second order peak is visible only with deuterated oil when the incoherent background is low. These features are better resolved in SAXS data (Fig. 5). 

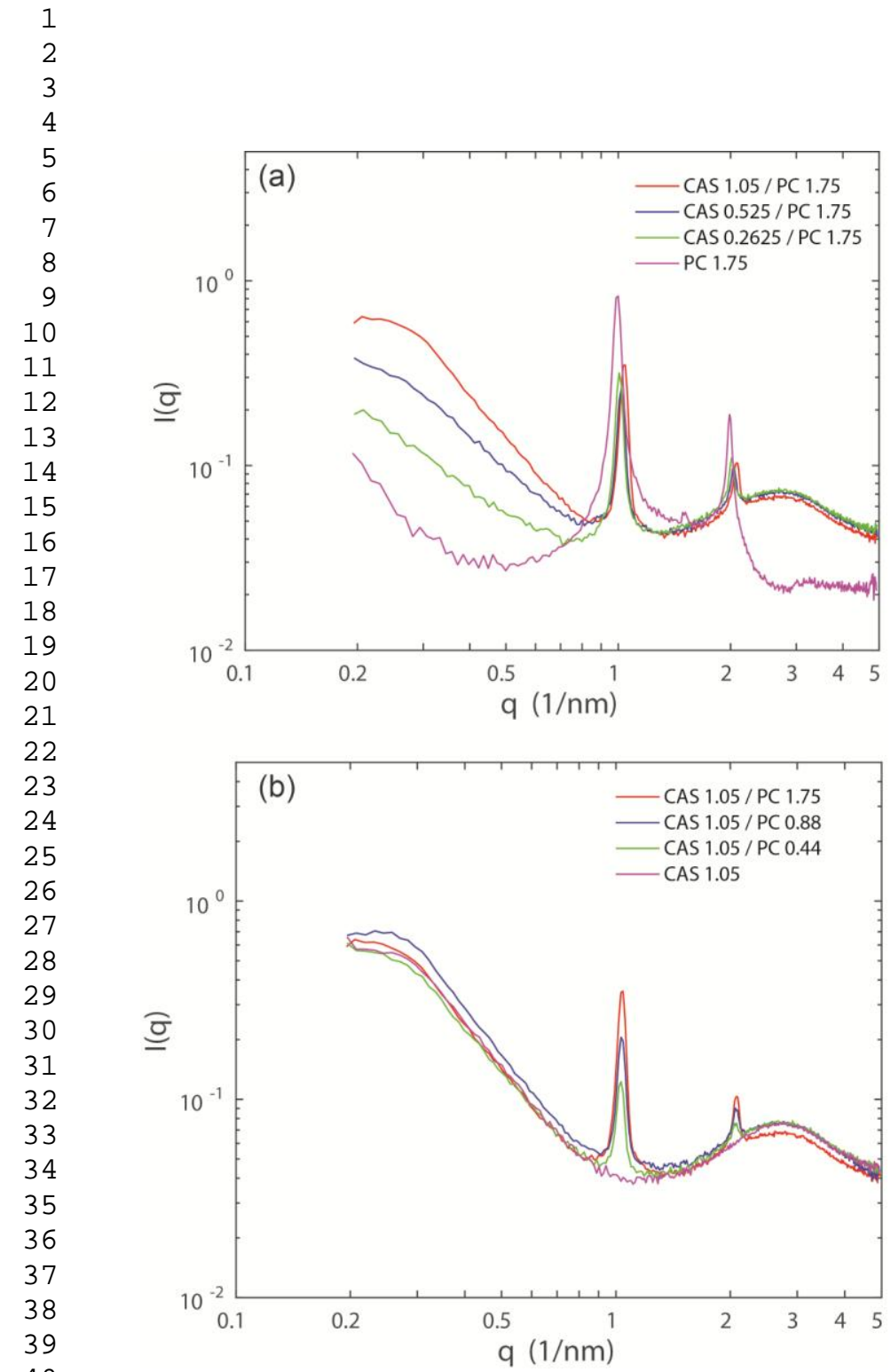

Fig. 5. (a) SAXS patterns of employed fish oil-in-water emulsions with various combinations of CAS $(0.2-1.05 \%)$ and PC (1.75\%) concentration, (b) SAXS patterns of employed fish oil-in-water emulsions with constant CAS fraction (1.05\%) and various PC concentrations (0.44-1.75\%). All material fractions are given in \%, w/w. 


\section{Table 1.}

The long period $L_{p}$ for PC bilayers and the coherence length $\zeta$ for PC multilayers. Above: PC in water systems with increasing concentration of PC (estimated from the SAXS and SANS data). Below: PC bilayers in emulsions for various CAS/PC ratios (SAXS)

\begin{tabular}{|c|c|c|c|c|c|c|c|c|c|c|}
\hline $\mathrm{PC}$, conc. $(\%, \mathrm{w} / \mathrm{w})$ & 0.4 (SAXS) & 0.9 (SAXS) & \multicolumn{2}{|c|}{0.4 (SANS) } & \multicolumn{2}{|c|}{0.9 (SANS) } & \multicolumn{2}{|c|}{1.8 (SANS) } & \multicolumn{2}{|c|}{6 (SANS) } \\
\hline$L_{\mathrm{p}}(\mathrm{nm})$ & $6.29 \pm 0.01$ & $6.33 \pm 0.01$ & $6.22 \pm 0.0$ & & $6.28 \pm 0$ & .08 & $6.35=$ & \pm 0.08 & 6.35 & \pm 0.08 \\
\hline$\xi(\mathrm{nm})$ & $97.5 \pm 0.2$ & $87.5 \pm 0.1$ & $47 \pm 1$ & & $40 \pm 1$ & & $40 \pm 1$ & & $40 \pm$ & \\
\hline CAS/PC (\%, w/w) & $1.05 / 1.75$ & $-/ 2.8$ & $1.05 / 0.88$ & 1.05 & /0.44 & 0.525 & $/ 1.75$ & $0.263 /$ & 1.75 & $-/ 1.75$ \\
\hline$L_{\mathrm{p}}(\mathrm{nm})$ & $6.06 \pm 0.01$ & $6.33 \pm 0.01$ & $6.06 \pm 0.01$ & 6.13 & \pm 0.01 & $6.17 \pm$ & 0.01 & $6.25 \pm 0$ & .01 & $6.32 \pm 0.01$ \\
\hline$\xi(\mathrm{nm})$ & $110.6 \pm 0.2$ & $88.4 \pm 0.1$ & $108.4 \pm 0.1$ & 107. & $7 \pm 0.1$ & 110.9 & \pm 0.1 & $120.0 \pm$ & -0.2 & $116.4 \pm 0.1$ \\
\hline
\end{tabular}

Fig. 5 plots the SAXS patterns for oil-in-water emulsion with the constant PC concentration and increasing CAS concentration as well as with constant CAS concentration and increasing PC concentration. In order to magnify the scattering contribution of $\mathrm{PC}$, the latter curves where the scattering contribution of CAS is subtracted are shown in Fig. S5 in the Supplementary Material. The data are dominated by Bragg reflections arising from PC. The long period for PC bilayers and the coherence length $\xi$ for the PC multilayers in emulsions for various CAS/PC ratios estimated from these reflections are compiled in Table 1 . This means that $\zeta$ describes the order within the PC layers and not the overall size of PC particles.

The location of the PC peak shifted significantly to the higher $q$ values with the increasing concentration of CAS (0 to 1.05\%) (Fig. 5a). The reason for this is that the periodic repeat distance of the PC bilayers decreased, which could be due to either decrease in the water amount in between 2 PC bilayers or increased order. This change in the overall arrangement of the bilayer stacking could be due to the interaction between CAS and PC at the oil-water interface. 
The packing of PC molecules may be influenced by the presence of CAS. It was suggested by Fang and Dalgleish that a complex between DOPC and $\beta$-casein was formed involving the hydrophobic domains of the two components, whereas it was not evident for DOPC and $\alpha_{s 1}$-casein, which makes the removal of casein from the interfacial region highly specific [35].

The CAS contribution increases with increasing CAS concentration (Fig. 5a). From the phase considerations (see section 3.1) we estimate that about 20\% CAS remains free in the water phase. The rest remains bound to the interface but we cannot say how strong this binding is. The fact that PC peaks are proportional to PC concentration implies that there is no significant interaction between CAS and PC located in the multilayers in the bulk phase. However, the multilayers become larger in emulsions, which points to increased interactions or a confinement effect between droplets.

In the case of emulsion, we need to consider both free CAS, which presumably is in the micellar form, and additionally a thin surface of PC and CAS formed at the oil-water interface. We analyzed the micellar CAS using the same fitting procedure as for pure CAS solution and the value obtained for $\eta$ were taken as measure of the amount of micellar CAS in the water phase. Thus, if the $\eta$ value in emulsion is the same as, say in 3\% CAS solution, then the weight fraction of the micellar CAS in the water phase is assumed to be $3 \%$ and the intensity due to micellar CAS then $I_{m c}=\varphi_{w} I_{3 \%}$, where $\varphi_{w}$ is the water volume fraction in emulsion and $I_{3 \%}$ is the intensity in the pure CAS water system. In general, the intensity was a linear interpolation between two closest pure CAS measurements. 


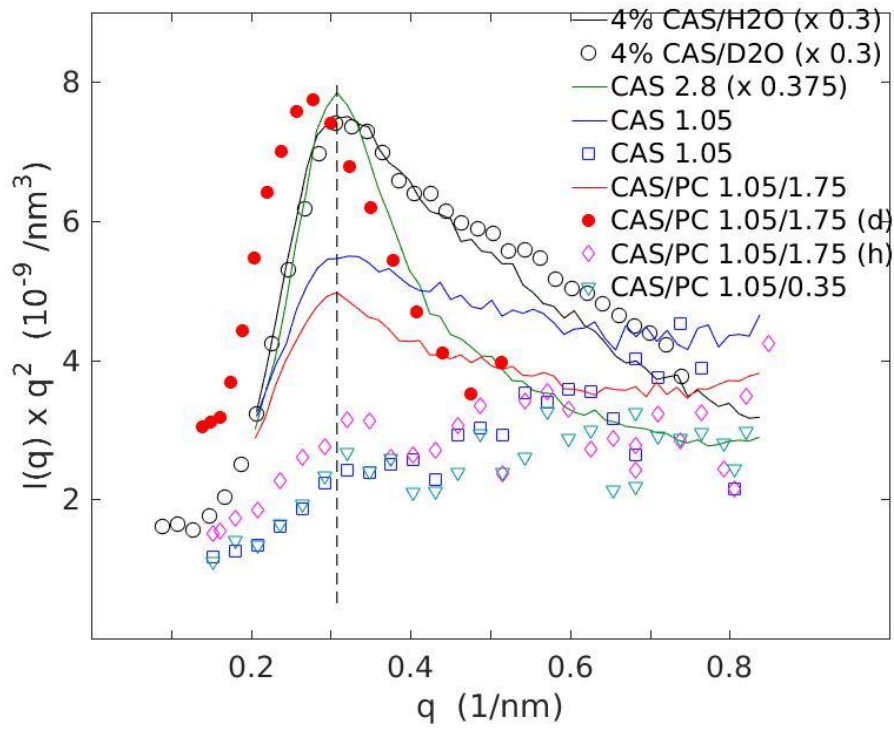

Fig. 6. SAXS (solid lines) PC $1.75 \%$ emulsion subtracted from CAS $1.05 \%+$ PC $1.75 \%$, SANS (circles) subtracted contribution from the PC monolayer as a power law background, and SANS (diamonds) CAS 4\%. All material fractions are given in $\%, \mathrm{w} / \mathrm{w}$.

Fig. 6 shows the comparison between the broad CAS peak as seen in emulsion by SANS and SAXS. The peak position for CAS in water is shown for comparison. The contribution from the proposed PC monolayer is subtracted and the intensity curves are normalized to the same water phase fractions and CAS/water scattering contrast. This comparison shows how the peak moves to the smaller scattering angles in emulsion and at the same time the peak intensity drops.

The SAXS curves show very slight decrease in peak position (increase in particle distance) and both the intensity level and peak width (related to the P-Y volume fraction, $\eta$ ) corresponds to $40-60 \%$ CAS in particles. Since the amount of free CAS is $20 \%$ at maximum (Table S6, the Supplementary Material), the SAXS data implies that either there is a time dependence to this amount or the adsorbed CAS exhibits similar inter-particle interference effects as free CAS. 
The SANS measurements in fish oil (open symbols) typically showed a weak maximum at the same position as in the original CAS in water system. The width of the peak is difficult to establish, but the intensity is consistent with $20 \%$ amount of free CAS. Thus, the adsorbed CAS seemingly does not contribute to scattering at this $q$-range.

The SANS measurement in deuterated hexadecane emulsions (solid symbols) showed significantly larger intensity and the characteristic distance between CAS particles is increased from $20.4 \mathrm{~nm}$ for the CAS in water up to $26 \mathrm{~nm}$ for CAS in emulsion (Fig. 6).

This means that even though CAS particles were not tightly packed on the interface forming a welldefined film (the interface is rather dominated by a well-defined PC monolayer), the CAS particles remain loosely bound and influenced by its vicinity forming supposedly a patchy array on it. This observation should be compared to the studies that have shown surfactants replaced proteins adsorbed at the oil-water interface. This phenomenon was related to the type of the surfactant, e.g., hydrophiliclipophilic balance, number of alkyl chains attached to the molecule, as well as the ways of surfactant incorporation during emulsion production, such as addition of the surfactant before or after homogenization $[17,18]$. Previous studies reported that Tween 60 replaced all the protein adsorbed at the oil-water interface. However, PC did not act in the same way, but instead interacted with protein at the interface and formed a mixed interfacial layer $[15,36]$. 
Fig. 7 outlines qualitative interpretation of our results. A PC monolayer separates oil and water phases. 80\% CAS particles are influenced or loosely bound to the interface but are not forming continuous coverage and true 2-dimensional system. The distance between aggregated CAS particles is increased compared to CAS aggregates in pure CAS in water system. PC multilayers become larger in the presence of interface compared to the pure PC in water. 


\section{Conclusions}

This study enlightens the interfacial structure of high fat (70\%) fish oil-in-water emulsion and provides information of the interaction and adsorption behavior of the combined use of CAS and PC emulsifiers. CAS is suggested to form thicker but less dense interface while a PC monolayer (3.2 nm thick) separates oil and water phases also filling the gaps in between CAS molecules. $80 \%$ CAS particles are influenced or loosely bound to the interface but are not forming continuous coverage and true 2dimensional system. The distance between aggregated CAS particles was increased compared to CAS aggregates in pure CAS in water system. PC multilayers become larger in the presence of the interface compared to the pure PC mixtures and bilayers become larger with increasing PC concentration.

By providing a direct structural interpretation, this study has implications in those food applications where the combination of emulsifiers is used to improve oxidative stability (see, e.g., $[11,12,13,14]$ ). It may also have implications in pharmaceutical applications as it relates to the intravenous lipid emulsions. Further research will concern different emulsion systems produced with various emulsifier types. The future work will also focus on the contrast variation studies of bulk phases. This would provide exact information of how the emulsifier fractions are distributed between interface and bulk.

\section{Acknowledgements}

We thank Lipoid GmbH, Arla Foods Ingredients amba and Vesterålens for providing the emulsifiers and cod liver oil, and the Danish Council for Independent Research for financing the project Mapping and Characterizing of Lipid Oxidation in Emulsified Systems (MAPOX, DFF-4184-0123A).

\section{Appendix A. Supplementary Material}




\section{References}

[1] J. Allaire, P. Couture, M. Leclerc, A. Charest, J. Marin, M.-C. Lépine, D. Talbot, A. Tchernof, B. Lamarche, Am. J. Clin. Nutr., 104 (2016) 280-287.

[2] T. Wysoczański, E. Sokoła-Wysoczańska, J. Pékala, S. Lochyński, K. Czyz, R. Bodkowski, G. Herbinger, B. Patkowska-Sokoła, T. Librowski, Curr. Med. Chem., 23-8 (2016) 816-831.

[3] P. D. Nichols, A. McManus, K. Krail, A. J. Sinclair, M. Miller, Nutrients, 6 (2014) 3727-3733.

[4] C. Song, C. Shieh, Y. Wu, A. Kalueff, S. Gaikwad, K. Su. Prog. Lipid Res., 62 (2016) 41-54.

[5] B. Yesiltas, P. J. García-Moreno, A.-D. M. Sørensen, C. Jacobsen. Eur. J. Lipid Sci. Technol., 119 (2017) 1600484.

[6] B. Yesiltas, P. J. García-Moreno, A.-D. M. Sørensen, S. Anankanbil, Z. Guo, C. Jacobsen, J. Agric. Food Chem.,66 (2018) 12512-12520..

[7] A. F. Horn, N. S. Nielsen, C. Jacobsen, Int. J. Food Sci. Technol., 47 (2012) 1097-1108.

[8] C. Jacobsen, M. B. Let, N. S. Nielsen, A. S. Meyer, Trends Food Sci. Technol., 19 (2008) 76-93.

[9] C. C. Berton-Carabin, M. H. Ropers, C. Genot, Compr. Rev. Food Sci. Food Saf., 13 (2014) 945-977.

[10] C. Berton, M.-H. Ropers, M. Viau, C. Genot, J. Agric. Food Chem., 59, (2011) 5052-5061.

[11] E. Dickinson, Colloids Surf. B, 20 (2001) 197-210.

[12] Y. Fang, D. G. Dalgleish, J. Col. Interface Sci., 156 (1993) 329-334.

[13] D. G. Dalgleish, M. Srinivasan, H. Singh, J. Agric. Food Chem., 43 (1995) 2351-2355. 
[14] R. Waninge, P. Walstra, J. Bastiaans, H. Nieuwenhuijse, T. Nylander, M. Paulsson, N. Bergenståhl, J. Agric. Food Chem., 53 (2005) 716-724.

[15] Y. Fang, D. G. Dalgleish, J. Am. Oil. Chem. Soc., 73(4) (1996) 437-442.

[16] D. G. Dalgleish, Trends Food Sci. Technol., 8 (1997) 1-6.

[17] D. J. McClements, E. Decker, J. Agric. Food Chem., 66 (2018) 20-35.

[18] C. C. Berton-Carabin, L. Sagis, \& K., Schroën, Annu. Rev. Food Sci. Technol., 9, (2018) 24.124.37.

[19] M. B. Munk, F. H. Larsen, F. W. J. van den Berg, J. C. Knudsen, J. C., M. L. Andersen, Langmuir, 30, (2014) 8687-8696.

[20] P. H. Stothart, D. J. Cebula, J. Mol. Biol., 160 (1982) 391-395.

[21] T. F. Kumosinski, H. Pessen, H. M. Farrell, H. Brumberger, Arch. Biochem. Biophys, 266 (1988) $548-61$.

[22] C. G. de Kruif, T. Huppertz, V. S. Urban, A. V. Petukhov, Adv. Coll. Interface Sci., 171-172 (2012) 36-52.

[23] V. Rampon, A. Riaublanc, M. Anton, C. Genot, D. J. McClements, J. Agric. Food Chem., 51, (2003) 5009-5905.

[24] P. J. García-Moreno, A. F. Horn, C. Jacobsen, J. Agric. Food Chem., 62, (2014) 1142-1152.

[25] C. Jacobsen, A. S. Meyer, J. Adler-Nissen, J.Food Lipids, 5, (1998) 87-101.

[26] A. Wacha, Z. Varga, A. Bóta, J. Appl. Cryst., 47, (2014) 1749-1754.

[27] A. Wacha, J. Appl. Cryst., 48, (2015) 1843-1848. 
[28] J. K. Percus, G. J. Yevick, J. Phys. Rev., 110 (1958) 1-13.

[29] A. Smialowska, L. Matia-Merino, B. Ingham, A. J. Carr, Colloids Surf. A, 522 (2017) 113-123.

[30] P. Thomar, T. Nicolai, Food Hydrocoll., 49 (2015) 224-231.

[31] D. Farrer, A. Lips, Int. Dairy J., 9 (1999) 281-286.

[32] T. Huppertz, I. Gazi, H. Luyten, H. Nieuwenhuijse, A. Alting, E. Schokker, Int. Dairy J., 74 (2017) 1-11.

[33] T. Huppertz, P.F. Fox, A.L. Kelly, in Rickey Y. Yada (Eds.), Proteins in Food Processing, Vol.2, Woodhead Publishing, Cambridge, 2018, Chapter 3.

[34] P. Balgavý, M. Dubnicková, N. Kucerka, M. A. Kiselev, S. P. Yaradaikin, D. Uhríková, Biochim. Biophys. Acta, 1512 (2001) 40-52.

[35] Y. Fang, D. G. Dalgleish, in E. Dickinson, D. Bergenståhl (Eds.), Food Colloids Protein, Lipids and Polysaccharides, Woodhead Publishing, Cambridge, 2004.

[36] I. Heertje, H. van Aalst, J. C. G. Blonk, A. Don, J. Nederlof, E. H. Lucassen-Reynders, Lebensm.Wiss. u.Technol., 29 (1996) 217-226. 
PC caseinate water

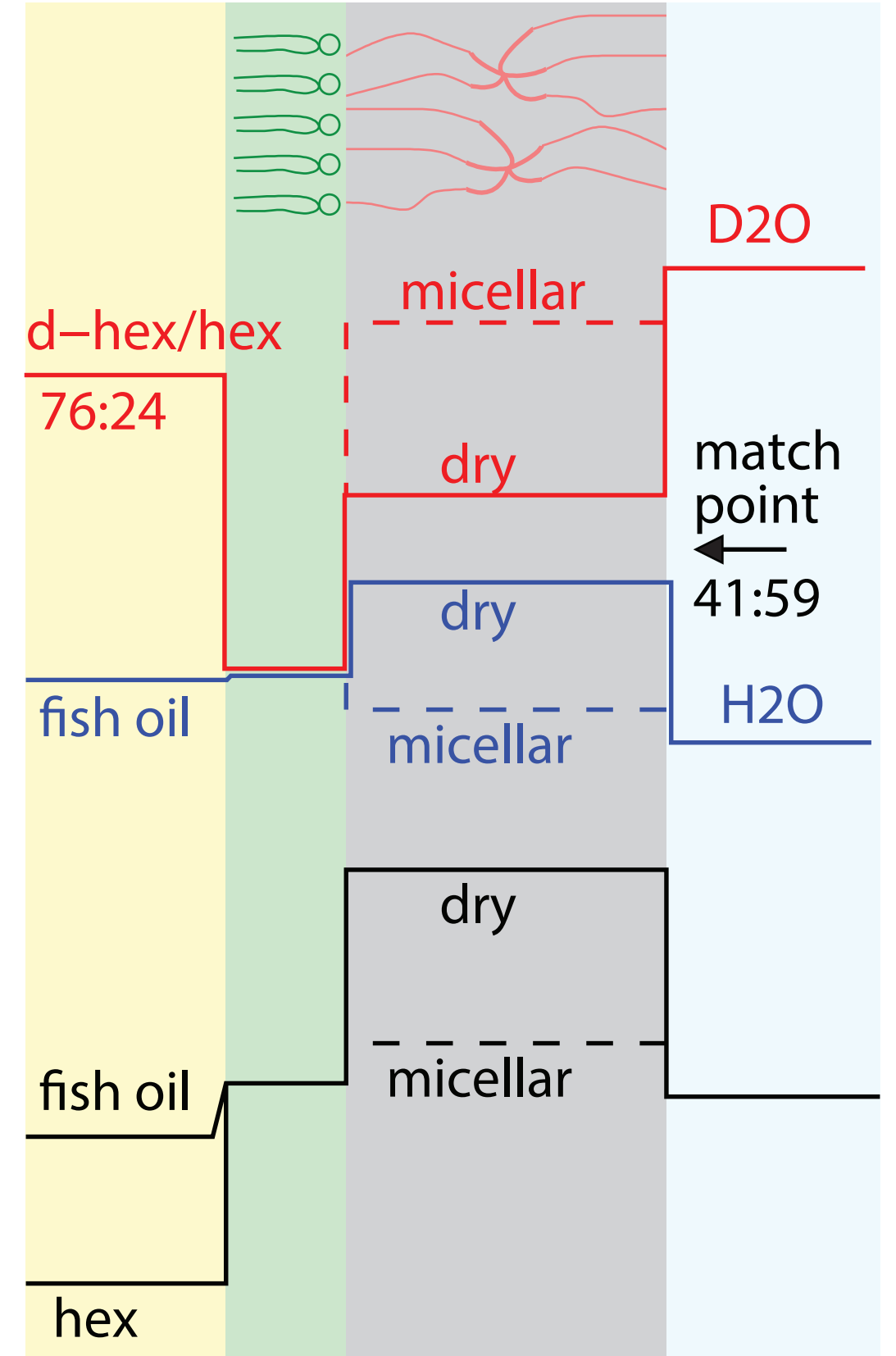

(b)

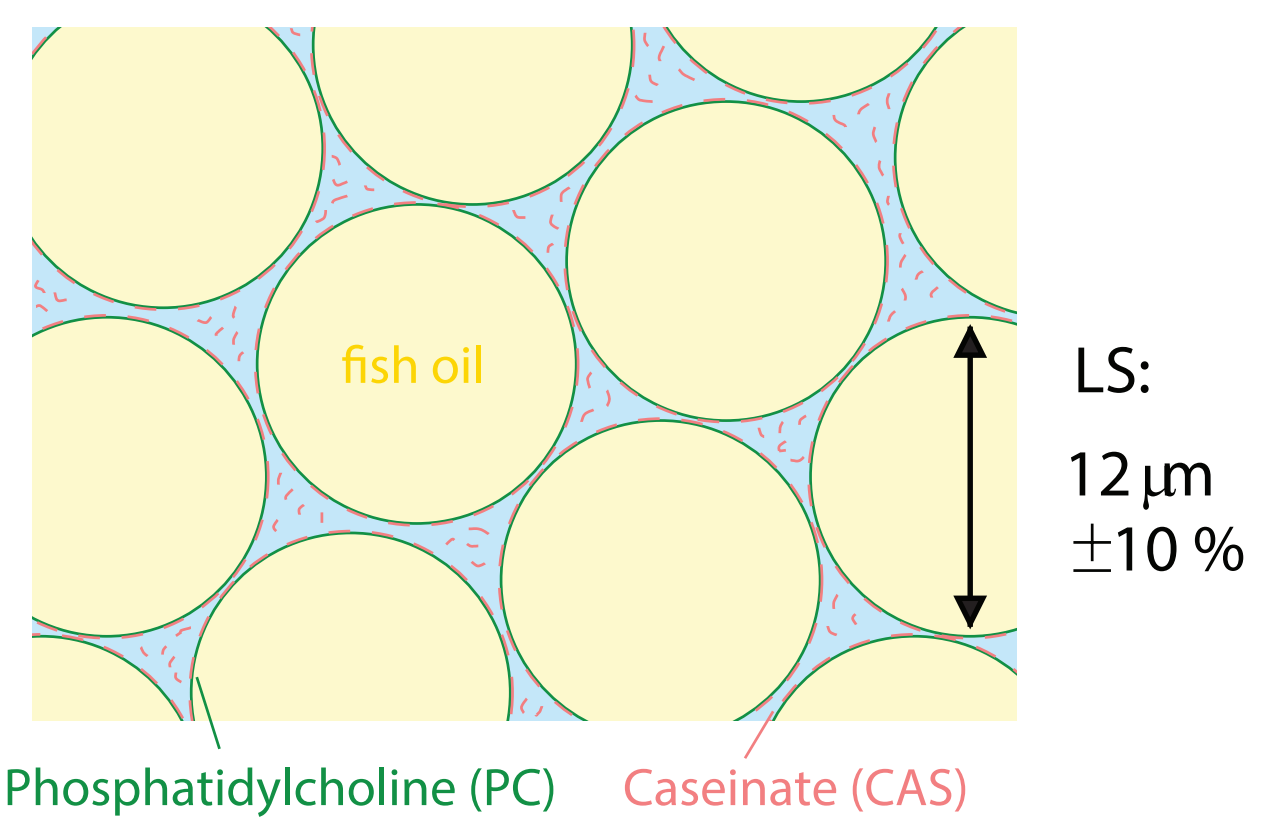

Phosphatidylcholine (PC) Caseinate (CAS) 

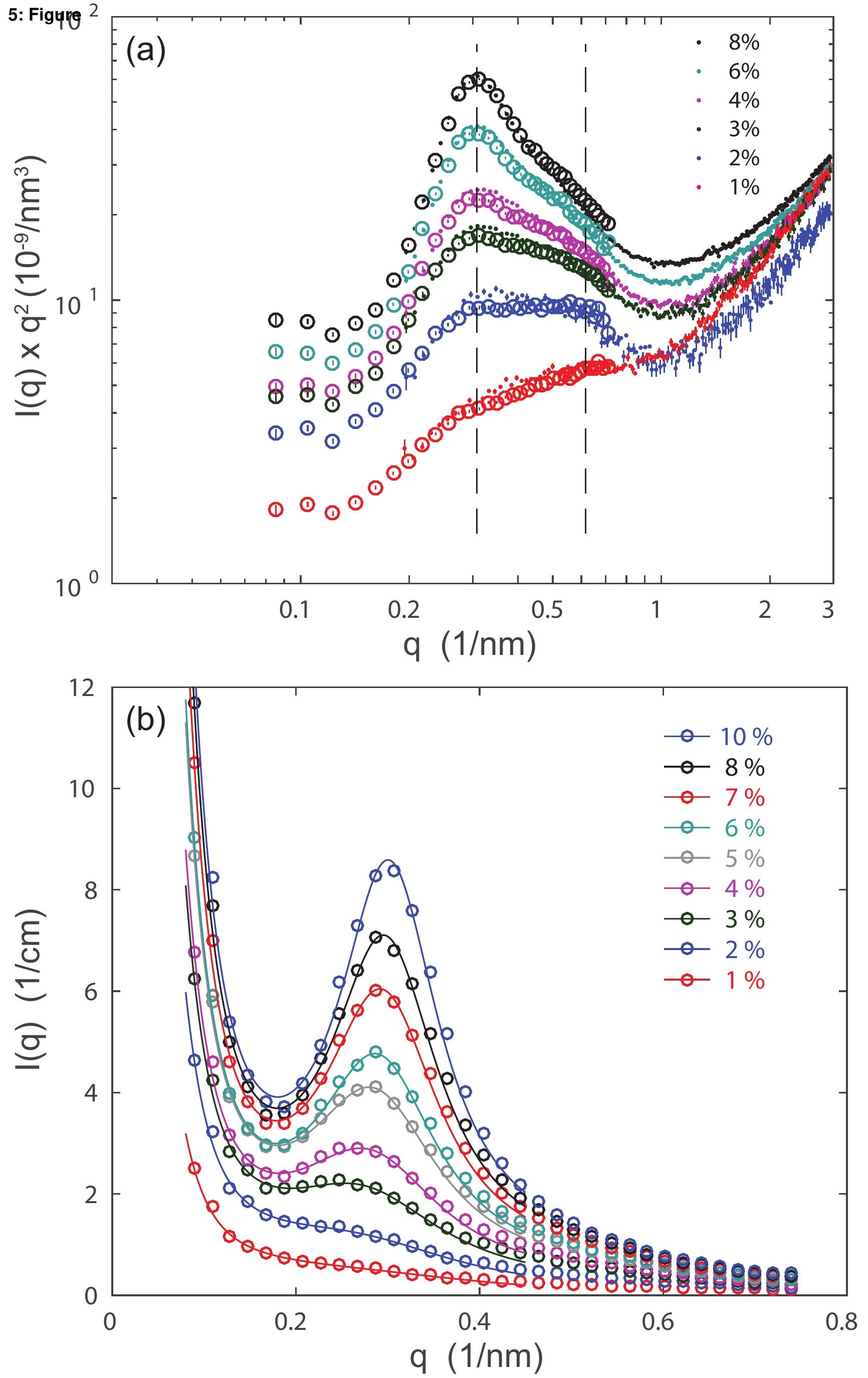
hydrophobic core
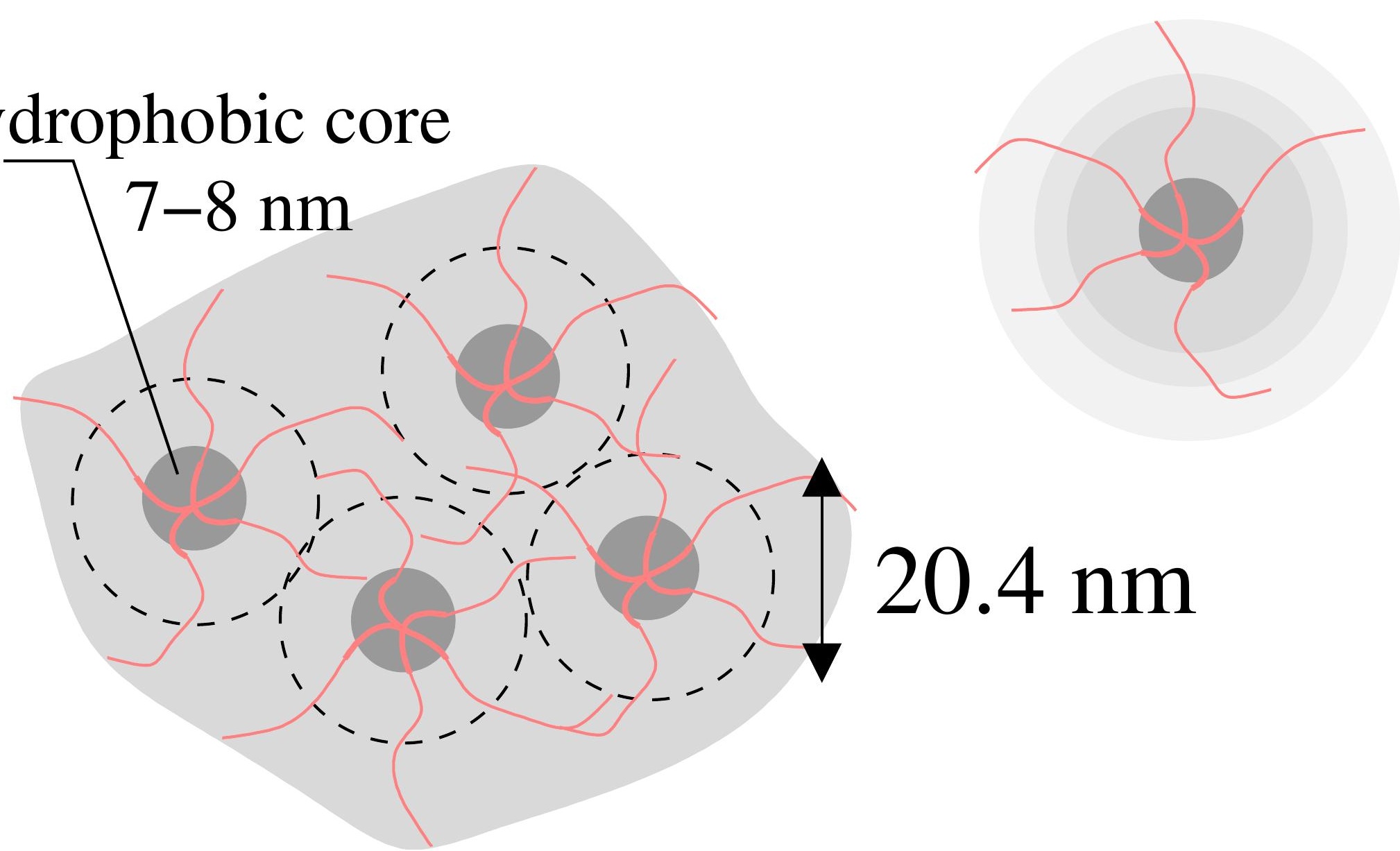

$20.4 \mathrm{~nm}$ 


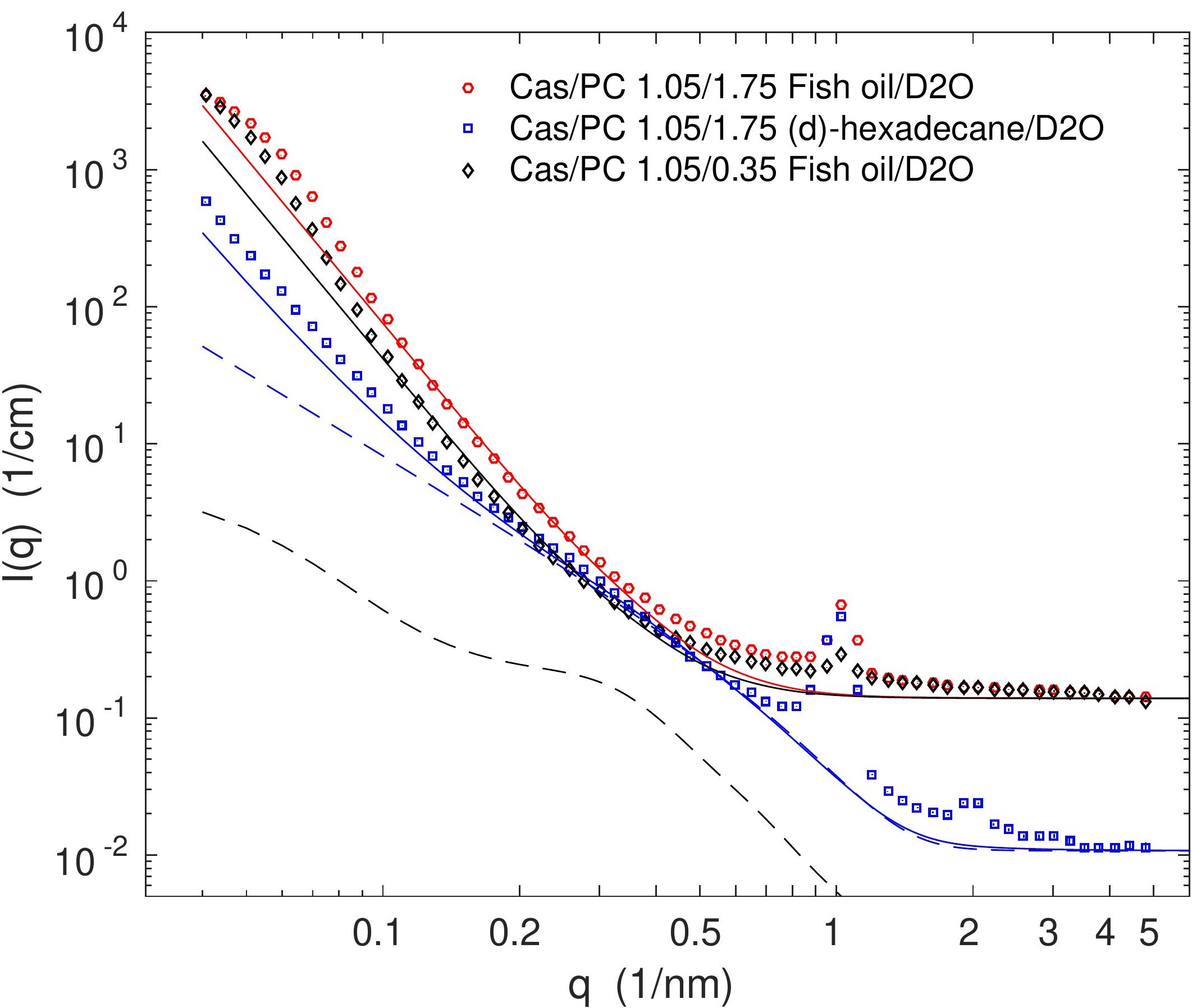




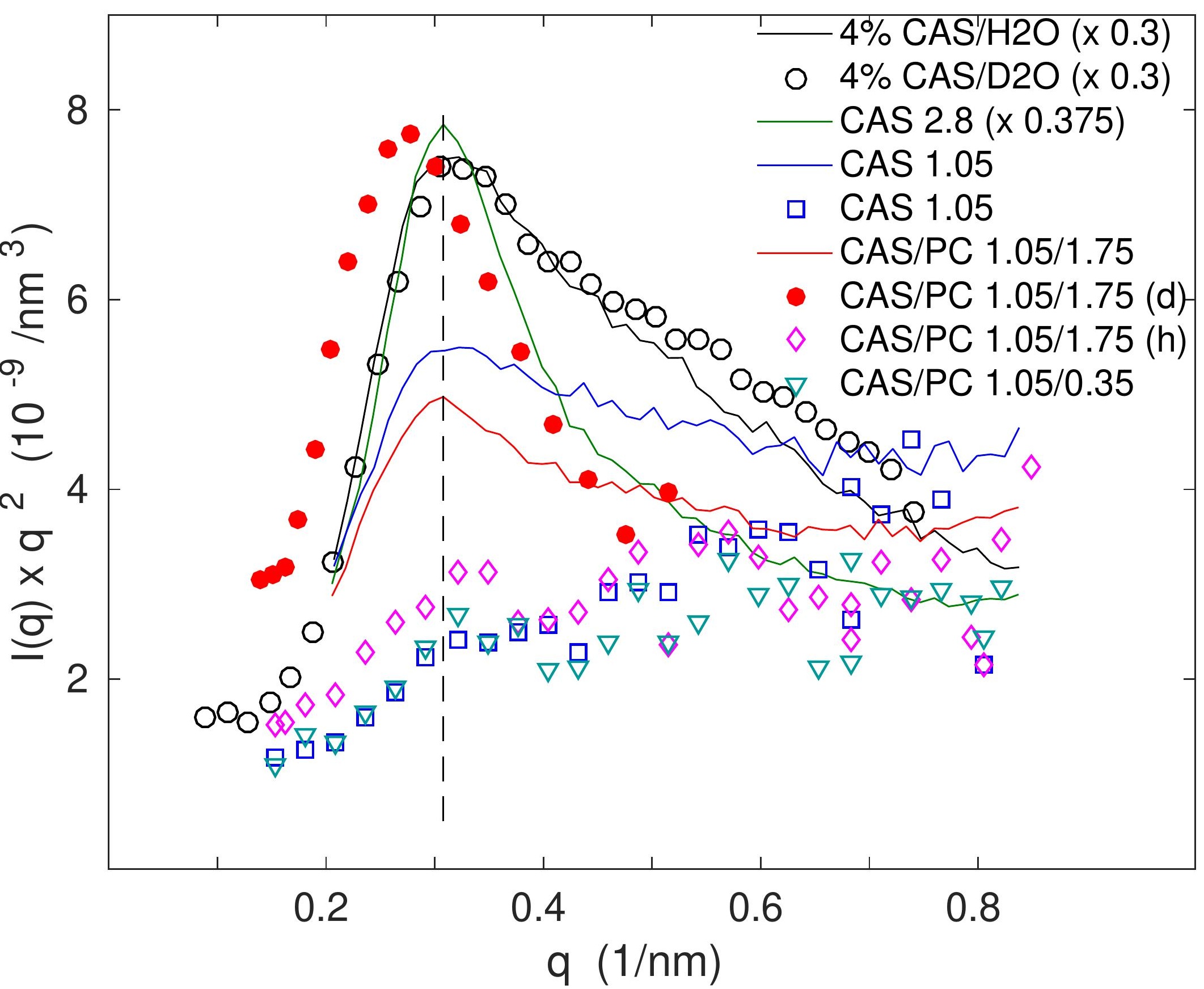




\section{5: Figure}

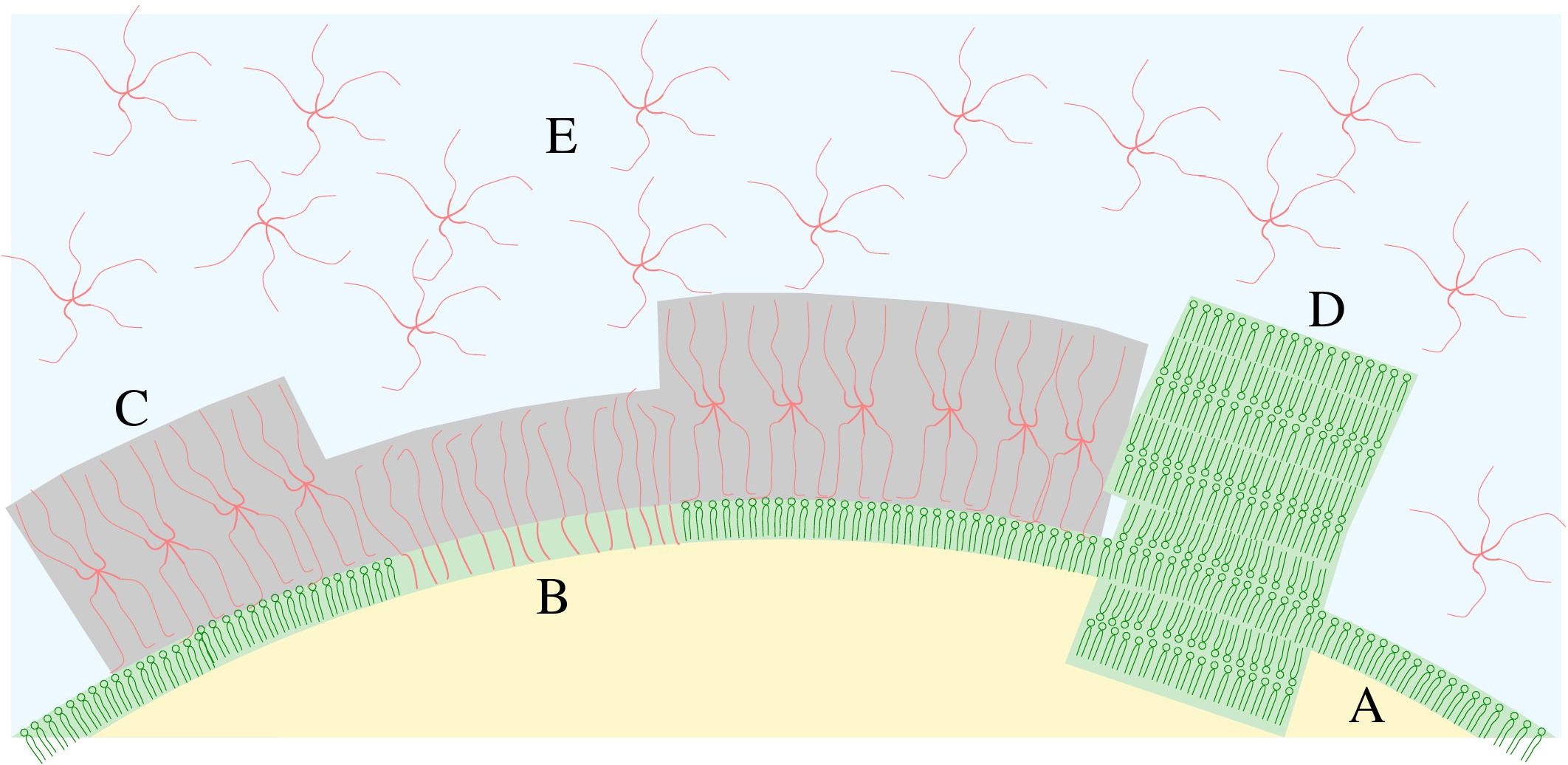




\section{Table 1.}

The long period $L_{p}$ for PC bilayers and the coherence length $\zeta$ for PC multilayers. Above: PC in water systems with increasing concentration of PC (estimated from the SAXS and SANS data). Below: PC bilayers in emulsions for various CAS/PC ratios (SAXS)

\begin{tabular}{lllllll}
\hline PC, conc. $(\%, w / w)$ & 0.4 (SAXS) & 0.9 (SAXS) & 0.4 (SANS) & 0.9 (SANS) & 1.8 (SANS) & 6 (SANS) \\
\hline$L_{\mathrm{p}}(\mathrm{nm})$ & $6.29 \pm 0.01$ & $6.33 \pm 0.01$ & $6.22 \pm 0.08$ & $6.28 \pm 0.08$ & $6.35 \pm 0.08$ & $6.35 \pm 0.08$ \\
$\xi(\mathrm{nm})$ & $97.5 \pm 0.2$ & $87.5 \pm 0.1$ & $47 \pm 1$ & $40 \pm 1$ & $40 \pm 1$ & $40 \pm 1$ \\
\hline \multicolumn{7}{c}{} \\
\hline
\end{tabular}


